\title{
Positive feedback loops exacerbate the influence of superspreaders in disease transmission
}

Klara Wanelik ( $\sim$ klara.wanelik@zoo.ox.ac.uk)

University of Oxford https://orcid.org/0000-0003-1485-0340

Michael Begon

University of Liverpool https://orcid.org/0000-0003-1715-5327

Andy Fenton

University of Liverpool

Rachel Norman

University of Stirling

Pablo Beldomenico

Universidad Nacional del Litoral

Article

Keywords: Epidemic models, supershedders, supercontactors, COVID-19, R0, final epidemic size

Posted Date: January 17th, 2022

DOI: https://doi.org/10.21203/rs.3.rs-1171612/v1

License: (c) (i) This work is licensed under a Creative Commons Attribution 4.0 International License.

Read Full License 
1 Positive feedback loops exacerbate the influence of superspreaders in disease

2 transmission

3

4 Klara M. Wanelik², Mike Begon², Andy Fenton², Rachel A. Norman³, Pablo M.

$5 \quad$ Beldomenico $^{4}$

6

$7 \quad{ }^{1}$ Department of Zoology, University of Oxford, Oxford, UK.

$8 \quad 2$ Department of Evolution, Ecology and Behaviour, Institute of Infection, Veterinary and

9 Ecological Sciences, University of Liverpool, Liverpool, UK.

$10{ }^{3}$ Department of Computing Science and Mathematics, Faculty of Natural Sciences,

11 University of Stirling, Stirling, UK.

$12{ }^{4}$ Laboratorio de Ecología de Enfermedades, Instituto de Ciencias Veterinarias del

13 Litoral (Consejo de Investigaciones Científicas y Técnicas - Universidad Nacional del

14 Litoral), Esperanza, Argentina.

15

$16 *$ Corresponding author

17 Email: klara.wanelik@zoo.ox.ac.uk

18

19

20

21

22

23

24

25 


\section{$26 \underline{\text { Abstract }}$}

27 Superspreaders are recognised as being important drivers of disease spread, most 28 recently having played a major role in the ongoing COVID-19 pandemic. However, 29 models to date have assumed random occurrence of superspreaders, irrespective of 30 whom they were infected by. Evidence suggests though that this may not be the case, 31 and that those individuals infected by superspreaders may be more likely to become 32 superspreaders themselves, hence creating a positive feedback loop. This could happen, 33 for example, through superspreaders releasing high infective doses that cause high 34 infection intensities in newly-infected individuals, which increases the likelihood that 35 they, in turn, will become superspreaders. Here, we examine the effects of such a 36 positive feedback loop on (1) the final epidemic size, (2) the basic reproductive number, $R_{0}$, and (3) the peak prevalence of superspreaders. We show that positive feedback loops can have a profound effect on the outcome of an epidemic, even when the transmission advantage of superspreaders is moderate, and despite peak prevalence of superspreaders remaining low. We argue that positive superspreader feedback loops in different infectious diseases, including SARS-CoV-2, should be investigated further.

$\underline{\text { Keywords }}$

44 Epidemic models, supershedders, supercontactors, COVID-19, $R_{0}$, final epidemic size 


\section{Introduction}

Pathogen transmission requires an appropriate contact between a susceptible and infected individual, such that the susceptible individual is exposed to pathogens shed by the infected individual. For example, for the respiratory pathogen, SARS-CoV-2, this may include both direct and indirect contact: either physical contact or close proximity, which increases the risk of exposure to infective respiratory droplets, or indirect contact via infective aerosols suspended in the air or on objects contaminated with the pathogen by an infected person (Zhou et al., 2021). Most traditional compartmental models of infectious disease dynamics, for example SIR (Susceptible - Infected Recovered) models, assume that, on average, all individuals behave in the same way (Anderson \& May, 1981). However, it has long been known that some individuals, termed "superspreaders", contribute far more to pathogen transmission, and epidemic spread, than others (Anderson \& May, 1987, 1991; Woolhouse et al., 1997).

Superspreaders may be defined as those individuals who are in the upper $1 \%$ tail of the distribution of pathogen transmission (Lloyd-Smith et al., 2005), but the term has been more widely applied to those with a strongly disproportionate contribution. The importance of accounting for this individual variation in epidemiological models has more recently been recognised, not least because it has played a major role in the ongoing COVID-19 pandemic (Adam et al., 2020; Correa-Martínez et al., 2020; GómezCarballa et al., 2020; Liu et al., 2020; Miller et al., 2020; Zhang et al., 2020).

Superspreaders exist for most diseases, whether in human, livestock or wildlife populations (Stein, 2011), and may originate in two ways. First, supercontactors are those who transmit infection to more individuals because they have a larger number of 
contacts than the average. There are clear examples of this in the transmission of HIV and other sexually-transmitted diseases in humans, through those who are more sexually active (Aral, 2004); while for non-humans, the transmission of a hantavirus (Sin Nombre virus) in deer mice, for example, has been shown to be driven by animals with larger home ranges who engage in more territorial defence (Clay et al., 2009). Second, supershedders are individuals who shed more infectious particles than average and so increase the probability of infection once an appropriate contact has been made. In the case of HIV in humans, for example, individuals co-infected with other sexuallytransmitted diseases have been shown to shed more HIV (Cohen et al., 1996). In cattle, heterogeneity in shedding of Escherichia coli 0157 has also been described (Omisakin et al., 2003). Mathematically, both mechanisms for superspreading (supercontacting and supershedding) result in an increase in the transmission term in standard compartmental models, and an increase in the basic reproductive number $\left(R_{0}\right)$ of the pathogen (Lloyd-Smith et al., 2005), leading to an increased probability of establishment and rate of spread of the epidemic. However, Ma \& Earn (2006) showed that for a closed, fixed sized population, the formula for the final epidemic size is unchanged by the presence of superspreaders.

93

94 Standard models assume that the generation of superspreaders is random, irrespective of whom they were infected by (Ma \& Earn, 2006). Yet there is evidence from some systems of a positive feedback loop whereby those individuals infected by superspreaders are more likely to be superspreaders themselves. For example, supershedders could generate further supershedders if an individual exposed to a higher infection load is itself more likely to develop a higher infection load, because higher inoculum doses may be more likely to overwhelm the mechanisms of resistance, 
resulting in poor control of viral replication. Evidence for this comes from experiments

102 in various virus-host systems showing that the outcome of infection is dose dependent,

103 with animals receiving a higher dose developing more severe disease and more

104 pronounced viral replication and shedding than those receiving lower doses (Gaskell \&

105 Povey, 1979; Mumford et al., 1990; Strong et al., 2015; Zarkov \& Bochev, 2008). At the

106 individual level, infections arising from exposure to high doses of virus are more likely

107 to be symptomatic and to have higher intensity (Bjorkman et al., 2021; Dabisch et al.,

108 2021); whereas two independent studies found that asymptomatic, likely low viral load

109 cases pose a lower risk of transmission, with their infected contacts themselves more

110 likely to be asymptomatic (Ge et al., 2021; Wu et al., 2021). Such positive superspreader

111 feedback loops have recently been proposed as an explanation for the heterogeneous

112 propagation pattern of COVID-19 (Beldomenico, 2020; van Damme et al., 2021). SARS-

113 CoV-2 viral loads have been found to drive the size and duration of COVID-19 clusters

114 (Hay et al., 2021; Jones et al., 2021; Ladoy et al., 2021); while in a cohort study in Spain,

115 the viral load of index cases was a leading driver of SARS-CoV-2 transmission (Marks et

116 al., 2021). Moreover, the only empirical study that has so far explored the positive

117 feedback loop hypothesis, while controlling for number of secondary infections, found

118 that the ratio of observed to expected superspreader-superspreader dyads was greater

119 than that expected by chance in 13 of 18 infection trees (Taube et al., 2021).

121 Hence, despite the recognition that superspreaders may generate superspreaders, and

122 the suggestion that this may be important for diseases like COVID-19, the role it plays in

123 driving the epidemiology of such diseases remains unknown. In this study, therefore, we

124 develop a generic model to understand how such positive feedback loops affect (1) the 
125 final epidemic size, (2) the basic reproductive number, $R_{0}$, and (3) the peak prevalence 126 of superspreaders, of an emerging epidemic.

\section{$128 \quad$ Methods}

129 In order to understand the role of the positive feedback loop between superspreaders

130 we formulated the following model:

$$
\frac{d S}{d t}=-S \beta_{L}(L+p H)
$$

$$
\frac{d L}{d t}=S \beta_{L}\left(\left(1-\sigma_{L}\right) L+p\left(1-\sigma_{H}\right) H\right)-(\alpha+\gamma) L
$$

$$
\frac{d H}{d t}=S \beta_{L}\left(\sigma_{L} L+\sigma_{H} p H\right)-(\alpha+\gamma) H
$$

$$
\frac{d R}{d t}=\gamma(H+L)
$$

137 where $S$ refers to the number of susceptible hosts, $L$ to low-titre infected hosts (non-

138 superspreaders), $H$ to high-titre infecteds (superspreaders) and $R$ to recovereds. $\beta_{L}$

139 represents the baseline transmission rate arising from L-infected hosts. This baseline

140 rate is then increased through transmission by superspreaders (H-infected hosts) by a

141 magnitude $p$ (we assume $p \geq 1$ ) representing the factor by which the transmission rate

142 from a superspreader is greater than the transmission rate from a non-superspreader

143 (i.e., the transmission advantage of superspreaders); $p$ has no upper bound.

$145 \sigma_{L}$ is the proportion of infections from a non-superspreader that result in a

146 superspreader, and so represents background, spontaneous generation of

147 superspreaders that we might expect in the absence of positive feedback. $\sigma_{H}$ is the 
148 proportion of infections from a superspreader that result in another superspreader. $\sigma_{H}$

149 and $\sigma_{L}$ both vary between 0 and 1 , but if $\sigma_{H}>\sigma_{L}$ then superspreaders are more likely to

150 generate new superspreaders, and hence $\sigma_{H} / \sigma_{L}$ represents the strength of the positive

151 feedback loop of superspreader infections.

152

$153 \alpha$ is the death rate due to the disease, and $\gamma$ is the recovery rate from the disease, both

154 assumed here to be the same for superspreaders and non-superspreaders. For this

155 model, there are no natural births or deaths, so the population only changes in size due

156 to deaths resulting from the disease. So, if $\alpha=0$ then the population remains constant,

$157 \quad$ size $N=S+L+H+R$.

158

159 For an infection spreading through a closed, fixed sized population, it is possible to

160 calculate the final epidemic size - the total number of individuals infected throughout

161 the epidemic (Kermack \& McKendrick, 1927). Ma and Earn (2006) showed that the

162 formula for the final epidemic size, $Z$, is unchanged by the presence of a fixed proportion

163 of superspreaders:

164

165

$$
Z=S[0]\left(1-\operatorname{Exp}\left(-Z \frac{\beta_{L}}{\alpha+\gamma}\left(1-\sigma_{H}+p \sigma_{L}\right)-\left(\frac{\beta_{L}}{\gamma+\alpha}\right)(L[0]+p H[0])\right)\right)
$$

167 For a fixed population size of 1 and in the limit $I(0) \rightarrow 0, S(0) \rightarrow 1$ the final epidemic

168 size, $Z$, for all of the models they considered, is given by $Z=1-e^{-R_{0} Z}$ (Ma and Earn

169 2006). 
171 For the current model, when $\sigma_{H}=\sigma_{L}$ (i.e., when there is no positive feedback loop) the

172 final epidemic size, $Z$, is also given by the same formula (see Supplementary Materials).

173 However, when $\sigma_{H}>\sigma_{L}$, and so the positive feedback loop does exist, it is not possible

174 to use the methods of Ma and Earn to calculate the final epidemic size, since additional

175 non-linearities in this model disqualify the simplifications they use. However, in the

176 Supplementary Materials we show that in the presence of a transmission feedback loop

177 (i.e., when $\sigma_{H}>\sigma_{L}$ ), the final epidemic size will be larger than in the absence of any

178 such feedback loop, and this difference increases as the difference between $\sigma_{H}$ and $\sigma_{L}$

179 increases. In the results that follow, we quantify the final epidemic size numerically by

180 running models in R (R Core Team, 2020) using the deSolve package (Soetaert et al.,

1812010 ) and calculating the final epidemic size and the peak prevalence of

182 superspreaders, while varying the ratio $\sigma_{H} / \sigma_{L}$ (the strength of positive feedback loop,

183 assuming $\sigma_{H} / \sigma_{L} \geq 1$ ) and $p$ (the transmission advantage of superspreaders, assuming

$184 p \geq 1$ ). Other parameter values were kept constant and were chosen for illustrative

185 purposes (Table 1). The initial number of non-superspreader infecteds, $L$, was kept

186 constant at $10 \%$ of the total population size, $N$, as was the initial number of recovereds,

$187 R$, at $0 \%$. We varied the initial number of superspreaders, $S$, between $0-10 \%$. All models

188 were run for sufficient periods of time to ensure that the epidemic had completed in all 189 cases.

191 Furthermore, we calculated the basic reproductive number $\left(R_{0}\right)$, i.e. the number of new

192 infections generated by one infectious individual in a completely susceptible population

193 for each of these scenarios. Using the next generation method of Diekmann et al. (2010;

194 see the Supplementary Materials), $R_{0}$ for this model is given by: 


$$
R_{0}=\frac{N \beta_{L}\left(1+p \sigma_{H}-\sigma_{L}+\sqrt{\left(\sigma_{L}-1-p \sigma_{H}\right)^{2}-4 p\left(\sigma_{H}-\sigma_{L}\right)}\right)}{2(\alpha+\gamma)}
$$

198 Table 1 Model parameters and their values.

\begin{tabular}{|c|c|c|c|}
\hline Parameter & Symbol & $\begin{array}{l}\text { Per capita } \\
\text { rate }\end{array}$ & Comment \\
\hline Death rate due to disease & $\alpha$ & 0 & $\begin{array}{l}\text { For simplicity assumed no excess } \\
\text { death due to infection. }\end{array}$ \\
\hline Recovery rate & $\gamma$ & 0.1 & $\begin{array}{l}\text { Assumed an average infectious period } \\
\text { of one week. }\end{array}$ \\
\hline $\begin{array}{l}\text { Baseline transmission rate } \\
\text { arising from non- } \\
\text { superspreaders (L-infected } \\
\text { hosts) }\end{array}$ & $\beta_{L}$ & $8 \times 10^{-6}$ & $\begin{array}{l}\text { Used an arbitrary } \\
\beta_{L} \text { that put the baseline } R_{0} \text { in the } \\
\text { absence of superspreading (i.e., when } \\
p=1, \sigma_{H}=\sigma_{L} \text { ) below } 1 \text { (see } \\
\text { Supplementary Materials; for the } \\
\text { values used here, } R_{0}^{L}=0.8 \text { ). From this } \\
\text { basis we can explore whether } \\
\text { superspreading and/or a positive } \\
\text { feedback loop has the potential to } \\
\text { drive an epidemic that would not } \\
\text { otherwise occur. }\end{array}$ \\
\hline $\begin{array}{l}\text { Proportion of infections from a } \\
\text { non-superspreader (L-infected } \\
\text { host) that result in a } \\
\text { superspreader (H-infected } \\
\text { host) }\end{array}$ & $\sigma_{L}$ & 0.05 & $\begin{array}{l}\text { Assumed that } 5 \% \text { of infections from a } \\
\text { non-superspreader result in a } \\
\text { superspreader. }\end{array}$ \\
\hline $\begin{array}{l}\text { The number of times more } \\
\text { superspreaders (H-infected } \\
\text { hosts) generated by a } \\
\text { superspreader (H-infected } \\
\text { host) than a non- } \\
\text { superspreader (L-infected } \\
\text { host) i.e. the strength of the } \\
\text { positive feedback loop }\end{array}$ & $\sigma_{H} / \sigma_{L}$ & $1-20$ & $\begin{array}{l}\text { Varied from } 1 \text { (equal generation of } \\
\text { superspreaders by superspreaders } \\
\text { and non-superspreaders) to the } \\
\text { maximum feasible value of } 20 \text { ( } 20 \\
\text { times more superspreaders } \\
\text { generated by a superspreader than a } \\
\text { non-superspreader). This maximum } \\
\text { feasible value arises since } \sigma_{H} \text { cannot } \\
\text { exceed } 1 ; \text { given the default value of } \\
\sigma_{L}=0.05, \sigma_{H} / \sigma_{L} \text { cannot exceed } 20 \text {. }\end{array}$ \\
\hline $\begin{array}{l}\text { The number of times by which } \\
\text { the transmission rate from a } \\
\text { superspreader (H-infected } \\
\text { host) is greater than the } \\
\text { transmission rate from a non- } \\
\text { superspreader (L-infected } \\
\text { host; the transmission } \\
\text { advantage of superspreaders) }\end{array}$ & $p$ & $1-20$ & $\begin{array}{l}\text { Varied from } 1 \text { (equal transmission } \\
\text { rate from a superspreader and a non- } \\
\text { superspreader) to } 20 \text { ( } 20 \text { times } \\
\text { higher transmission rate from a } \\
\text { superspreader than from a non- } \\
\text { superspreader). }\end{array}$ \\
\hline
\end{tabular}


202 The final epidemic size (Fig. 1) and the basic reproductive number, $R_{0}$ (Fig. 2), both

203 increase with increases in the transmission advantage of superspreaders, $p$, and with

204 the strength of the positive feedback loop whereby superspreaders generate further

205 superspreaders, $\sigma_{H} / \sigma_{L}$. Hence both epidemic outcomes reach their highest values

206 when both $p$ and $\sigma_{H} / \sigma_{L}$ are high.

207

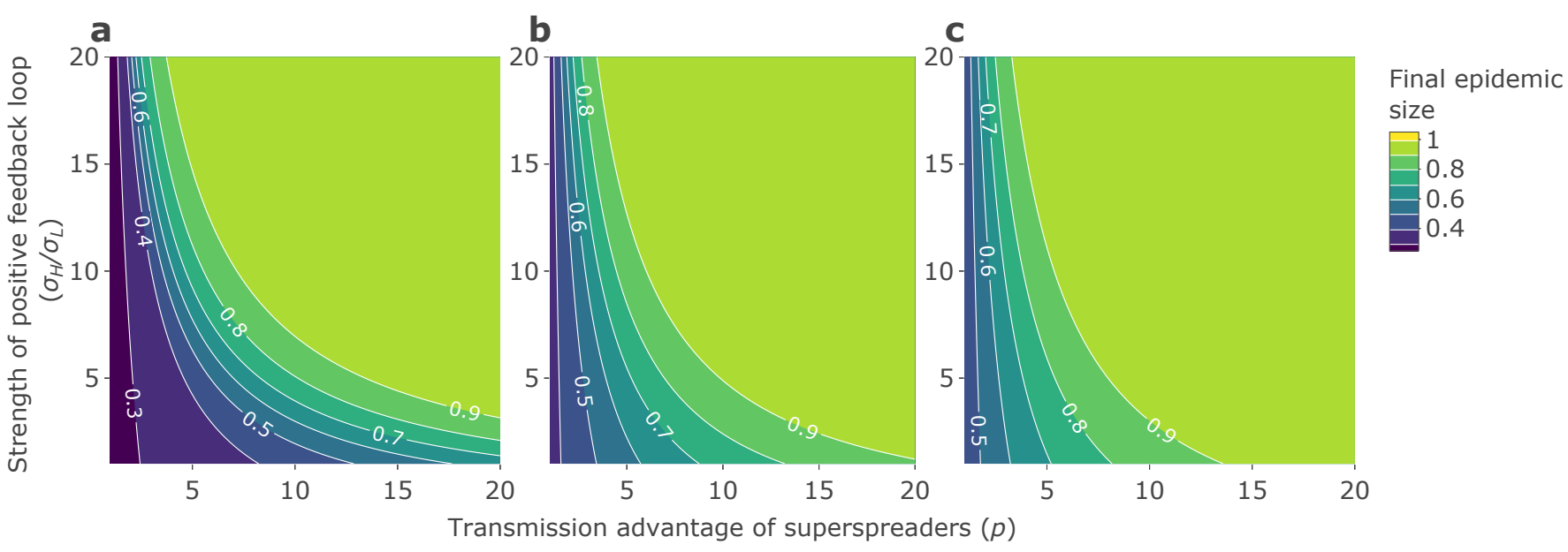

210 Fig. 1 Final epidemic size (where the maximum is 1, reflecting everyone in the

211 population becoming infected) as the strength of the positive feedback loop $\left(\sigma_{H} / \sigma_{L} ; \mathrm{y}\right.$ -

212 axis), the transmission advantage of superspreaders ( $p$; $x$-axis) and the initial number of

213 superspreaders (a: 0\%; b: 5\%; c: 10\%) are varied. See Table 1 for other parameter

214 values. 


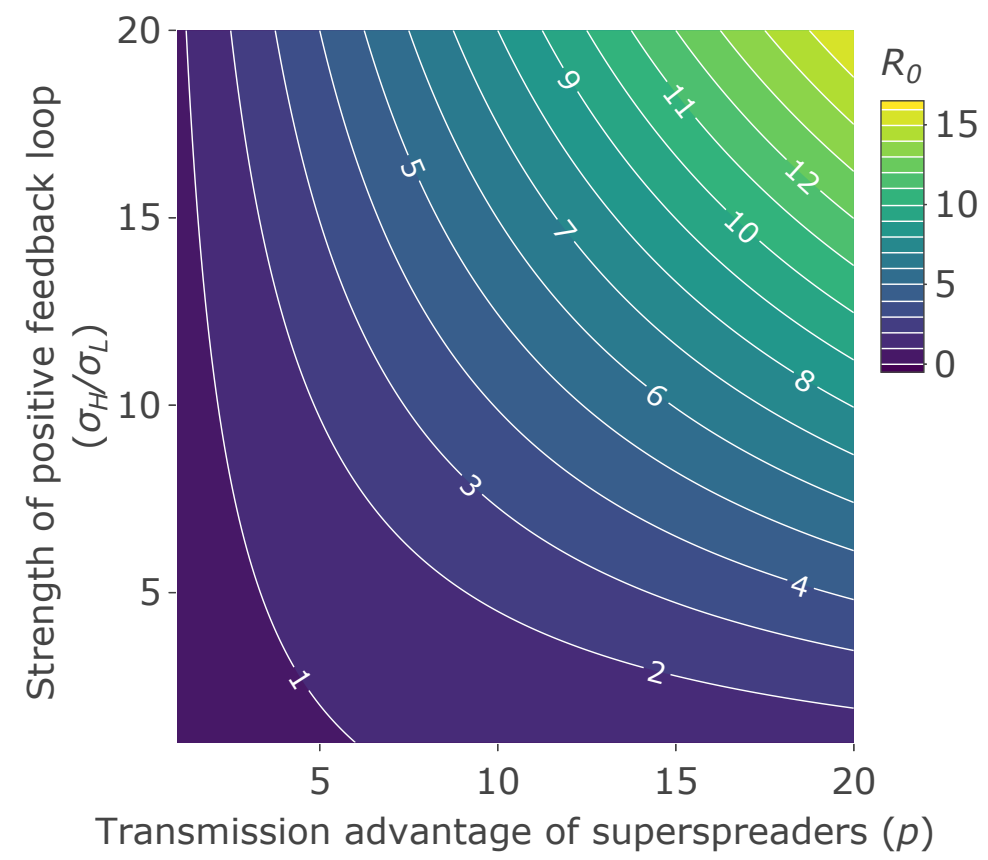

217

218 Fig. 2 The basic reproductive number $\left(R_{0}\right)$ as the strength of the positive feedback

$219 \operatorname{loop}\left(\sigma_{H} / \sigma_{L} ; \mathrm{y}\right.$-axis $)$ and the transmission advantage of superspreaders $(p ; \mathrm{x}$-axis $)$ are

220 varied. See Table 1 for other parameter values.

221

222 Of course, in the absence of superspreading $(p=1), R_{0}$ and the final epidemic size are

223 insensitive to $\sigma_{H} / \sigma_{L}$ since there is no superspreader advantage on which a feedback

224 loop might act (along the vertical axes of the figures). Indeed, as shown in the

225 Supplementary Materials, there is a lower threshold value of $p$, given by $\frac{(\alpha+\gamma)}{N \beta_{L}}$, below

226 which no viable amount of positive feedback can drive $R_{0}>1$ (for our baseline

227 parameter values, this lower threshold value of $p$ is 1.25 , suggesting superspreaders

228 need at least a $25 \%$ transmission advantage for an epidemic to occur, regardless of the

229 extent of positive feedback). Even marginally above this value, $R_{0}$ and the final epidemic

230 size are relatively insensitive to increases in the strength of the positive feedback loop

231 when the transmission advantage of superspreaders is low. Thus, for our baseline 
parameter values, feedback loop strength has little effect on outcome when superspreaders are, say, only twice as effective in transmission as non-superspreaders

$234(p=2)$, but the effect is profound as $p$ approaches 10 .

Conversely, in the absence of a feedback loop as assumed in previous studies $\left(\sigma_{H} / \sigma_{L}=\right.$

1 , along the horizontal axis in the figures) outcomes, especially $R_{0}$, may change little with superspreader advantage. For example, in the absence of feedback, and using the baseline parameter values to generate Fig. $2, R_{0}$ is less than 1 (the pathogen fails to cause an epidemic) unless $p$ exceeds 6 (see Supplementary Materials), and only reaches 1.6 as $p$ approaches 20 (the maximum illustrative value used here). But when there is

242 feedback, and especially when the feedback loop is strong (e.g. $\sigma_{H} / \sigma_{L}=20$ in Fig. 2) $R_{0}$ rises rapidly, reaching 16 in Fig. 2 as $p$ approaches 20 , and causing an epidemic $\left(R_{0}>1\right)$ with only very moderate levels of superspreader advantage ( $p \geq 1.25$; Supplementary Materials).

247 It is also noteworthy that this newly identified effect of the feedback loop on epidemic outcome can be profound even when the proportion of superspreaders in the population remains low. For example, at intermediate levels of superspreader advantage $(p=5)$, increasing feedback loop strength from 1 to 5 , an intermediate level of feedback, has a powerful effect on both final epidemic size (Fig. 1) and $R_{0}$ (Fig. 2), with $R_{0}$, for example, increasing from less than 1 (pathogen failing to cause an epidemic), to more than 1 (pathogen causing an epidemic). This is despite the peak prevalence of superspreaders not exceeding $\sim 0.5-10 \%$, depending on the initial number of superspreaders (Fig. 3). 


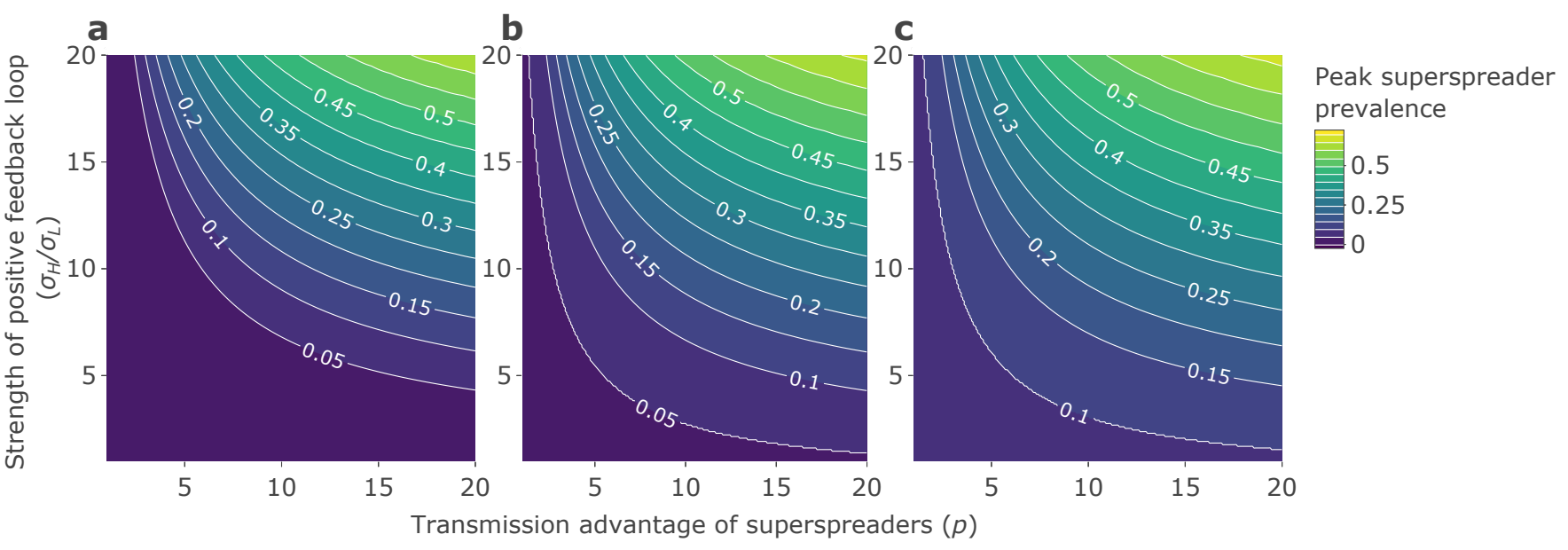

Fig. 3 Peak prevalence of superspreaders (as a proportion of total population size, $N$ ) as

260 the strength of the positive feedback loop $\left(\sigma_{H} / \sigma_{L} ; \mathrm{y}\right.$-axis), the transmission advantage of superspreaders ( $p$; x-axis) and the initial number of superspreaders (a: $0 \%$; b: $5 \%$; : 10\%) are varied. See Table 1 for other parameter values.

\section{Discussion}

Superspreaders are recognised as being important drivers of disease spread. However, models to date have assumed random generation of superspreaders, irrespective of whom they were infected by. Here, by contrast, and supported by a range of studies, we explore the importance of positive superspreader feedback loops, whereby those individuals infected by superspreaders are more likely to be superspreaders

271 themselves. We show that superspreaders in their own right may have little effect on

272 the outcome of an epidemic, but when positive superspreader feedback is included, the

273 effect may be profound, even when the transmission advantage of superspreaders is

274 moderate, and despite peak prevalence of superspreaders remaining low. Thus, our

275 theoretical framework explores the ideas proposed by Beldomenico (2020), whereby 
positive feedback loops have the potential to drive the heterogeneous propagation

pattern of a range of infectious diseases, potentially including SARS-CoV-2.

279 Positive feedback loops in superspreading could arise through both physiological and

280 behavioural mechanisms. There is evidence, elaborated above, that high infective doses

281 cause high infection intensity, which in turn expose new contacts to larger inoculum

282 sizes. By this means, supershedders may generate new supershedders (Beldomenico, 2020). Behaviourally, there is also some evidence that highly-connected individuals tend to contact other highly-connected individuals. In network theory, individuals (nodes) like this are described as having high eigenvalue centrality and have been shown to be important in epidemic spreading (Canright \& Engø-Monsen, 2006). In this case, the contact-status of those newly-infected individuals would not change upon transmission, so we cannot say that supercontactors generate new supercontactors. However, a disproportionate number of supercontactor-supercontactor dyads would still be observed. For example, in HIV transmission in humans, those who are more sexually active tend to infect others who are also more sexually active (Kelly et al., 2010); and for COVID-19, it has been suggested that those who are less likely to wear masks may be more likely to come into contact with, and so infect, others who equally do not wear masks and disregard public health recommendations generally (Taube et al., 2021). As such we would expect the overall consequences for the epidemiology of those systems to broadly follow those we present for our model.

In the context of COVID-19, and in addition to the supershedder and supercontactor processes described above, our model could also describe other scenarios. For example,

300 individuals infected by a new, highly-transmissable strain may represent 
superspreaders, who infect others with the same strain, therefore only generating more superspreaders $\left(\sigma_{H}=1\right)$; while the non-superspreaders are individuals infected by a

303 less transmissible strain, infecting others with the same strain and only generating more non-superspreaders $\left(\sigma_{L}=0\right)$. In a second scenario, if we assume that vaccines against SARS-CoV-2 are not entirely sterilising but reduce transmission potential (Gier et al., 2021; Singanayagam et al., 2021), then we can think of non-vaccinated individuals infected with the virus as being (relative) superspreaders, and the vaccinated as nonsuperspreaders. Behaviourally (culturally), there is likely to be some clustering, with the non-vaccinated superspreaders mostly interacting with other non-vaccinated individuals, thereby generating more superspreaders. Our model predictions suggest that such a scenario is likely to result in the non-vaccinated contributing more than previously recognised to the amplification of the epidemic.

314 It is noteworthy, too, that by introducing a positive feedback loop between

315 superspreaders, we inevitably introduce a positive feedback loop between nonsuperspreaders. Some positive feedback loops may therefore cause cascades of

317 superspreading, while others favour slow endemic circulation. This may help explain

318 why infectious diseases sometimes circulate at lower levels, while at other times there

319 are explosive uncontrollable outbreaks. The first year and a half of the COVID-19 pandemic in Vietnam, Thailand and Laos may be an example of the former, whereas the months that followed in those countries could be an example of the latter (Chookajorn et al., 2021).

324 Given the results from this initial study, we contend that the existence of positive

325 feedback loops in different host-pathogen systems should be investigated further. 


\section{Acknowledgements}

328 K.M.W. was supported by a European Research Council (ERC) research grant (No.

329 851550) while doing this work. R.A.N. was supported by The Leverhulme Trust,

330 fellowship RF-2019-331.

331

332 Author contributions

333 P.M.B. conceived the analysis. M.B., K.M.W., A.F. and R.A.N. designed the model. K.M.W.,

334 A.F. and R.A.N. analysed the data. K.M.W. led the writing of the manuscript. All authors

335 contributed critically to analysis discussion and manuscript drafts and approved a final

336 version for publication.

337

$338 \quad$ Competing interests

339 The authors declare no competing interests.

340

341

342

343

344

345

346

347

348

349

350

351 


\section{References}

Adam, D. C., Wu, P., Wong, J. Y., Lau, E. H. Y., Tsang, T. K., Cauchemez, S., Leung, G. M., \& Cowling, B. J. (2020). Clustering and superspreading potential of SARS-CoV-2 infections in Hong Kong. Nature Medicine, 26(11), 1714-1719. https://doi.org/10.1038/s41591-020-1092-0

Anderson, R., \& May, R. (1981). The population dynamics of microparasites and their invertebrate hosts. Philosophical Transactions of the Royal Society of London. B, Biological Sciences, 291(1054), 451-524. https://doi.org/10.1098/rstb.1981.0005

Anderson, R., \& May, R. (1987). Transmission dynainics of HIV infection. Nature, 326, $137-142$.

Anderson, R., \& May, R. (1991). Infectious Diseases of Humans: Dynamics and Control. Oxford University Press.

Aral, S. O. (2004). Sexual risk behaviour and infection: Epidemiological considerations. Sexually Transmitted Infections, 80(Suppl. 2), 8-12. https://doi.org/10.1136/sti.2004.011866

Beldomenico, P. M. (2020). Do superspreaders generate new superspreaders? A hypothesis to explain the propagation pattern of COVID-19. International Journal of Infectious Diseases, 96, 461-463. https://doi.org/10.1016/j.ijid.2020.05.025

Bjorkman, K. K., Saldi, T. K., Lasda, E., Bauer, L. C., Kovarik, J., Gonzales, P. K., Fink, M. R., Tat, K. L., Hager, C. R., Davis, J. C., Ozeroff, C. D., Brisson, G. R., Larremore, D. B., Leinwand, L. A., McQueen, M. B., \& Parker, R. (2021). Higher Viral Load Drives Infrequent Severe Acute Respiratory Syndrome Coronavirus 2 Transmission Between Asymptomatic Residence Hall Roommates. The Journal of Infectious Diseases, 224(8), 1316-1324. https://doi.org/10.1093/infdis/jiab386

Canright, G. S., \& Engø-Monsen, K. (2006). Spreading on networks: A topographic view. Complexus, 3(1-3), 131-146. https://doi.org/10.1159/000094195

Chookajorn, T., Kochakarn, T., Wilasang, C., Kotanan, N., \& Modchang, C. (2021). Southeast Asia is an emerging hotspot for COVID-19. Nature Medicine, 27, 14951496.

Clay, C. A., Lehmer, E. M., Previtali, A., St Jeor, S., \& Dearing, M. D. (2009). Contact heterogeneity in deer mice: Implications for Sin Nombre virus transmission. Proceedings of the Royal Society B: Biological Sciences, 276(1660), 1305-1312. https://doi.org/10.1098/rspb.2008.1693

Cohen, M. S., Hoffman, I. F., Royce, R. A., Kazembe, P., Dyer, J. R., Daly, C. C., Zimba, D., Vernazza, P. L., Maida, M., Fiscus, S. A., \& Eron, J. J. (1996). Reduction of concentration of HIV-1 in semen after treatment of urethritis: Implications for prevention of sexual transmission of HIV-1. Lancet, 349(9069), 1868-1873. https://doi.org/10.1016/S0140-6736(97)02190-9

Correa-Martínez, C. L., Kampmeier, S., Kümpers, P., Schwierzeck, V., Hennies, M., Hafezi, W., Kühn, J., Pavenstädt, H., Ludwig, S., \& Mellmann, A. (2020). A pandemic in times of global tourism: Superspreading and exportation of COVID-19 cases from a ski area in Austria. Journal of Clinical Microbiology, 58(6), 19-21. https://doi.org/10.1128/JCM.00588-20

Dabisch, P. A., Biryukov, J., Beck, K., Boydston, J. A., Sanjak, J. S., Herzog, A., Green, B., Williams, G., Yeager, J., Bohannon, J. K., Holland, B., Miller, D., Reese, A. L., Freeburger, D., Miller, S., Jenkins, T., Rippeon, S., Miller, J., Clarke, D., ... Hevey, M. (2021). Seroconversion and fever are dose-dependent in a nonhuman primate 
model of inhalational COVID-19. PLoS Pathogens, 17(8), 1-19. https://doi.org/10.1371/journal.ppat.1009865

Diekmann, O., Heesterbeek, J. A. P., \& Roberts, M. G. (2010). The construction of nextgeneration matrices for compartmental epidemic models. Journal of the Royal Society Interface, 7(47), 873-885. https://doi.org/10.1098/rsif.2009.0386

Gaskell, R. M., \& Povey, R. C. (1979). The dose response of cats to experimental infection with Feline Viral Rhinotracheitis virus. Journal of Comparative Pathology, 89(2), 179-191. https://doi.org/10.1016/0021-9975(79)90057-4

Ge, Y., Martinez, L., Sun, S., Chen, Z., Zhang, F., Li, F., Sun, W., Chen, E., Pan, J., Li, C., Sun, J., Handel, A., Ling, F., \& Shen, Y. (2021). COVID-19 Transmission Dynamics among Close Contacts of Index Patients with COVID-19: A Population-Based Cohort Study in Zhejiang Province, China. JAMA Internal Medicine, 181(10), 1343-1350. https://doi.org/10.1001/jamainternmed.2021.4686

Gier, B. de, Andeweg, S., Backer, J. A., RIVM COVID-19 surveillance and epidemiology team, Hahné, S. J. M., Hof, S. van den, Melker, H. E. de, \& Knol, M. J. (2021). Vaccine effectiveness against SARS-CoV-2 transmission to household contacts during dominance of Delta variant (B.1.617.2), August-September 2021, the Netherlands. MedRxiv, September, 2021.10.14.21264959. https://www.medrxiv.org/content/10.1101/2021.10.14.21264959v1\%0Ahttps:// www.medrxiv.org/content/10.1101/2021.10.14.21264959v1.abstract

Gómez-Carballa, A., Bello, X., Pardo-Seco, J., Martinón-Torres, F., \& Salas, A. (2020). Mapping genome variation of SARS-CoV-2 worldwide highlights the impact of COVID-19 super-spreaders. Genome Research, 30(10), 1434-1448. https://doi.org/10.1101/GR.266221.120

Hay, J. A., Kennedy-Shaffer, L., Kanjilal, S., Lennon, N. J., Gabriel, S. B., Lipsitch, M., \& Mina, M. J. (2021). Estimating epidemiologic dynamics from cross-sectional viral load distributions. Science, 373(6552). https://doi.org/10.1126/science.abh0635

Jones, T. C., Biele, G., Mühlemann, B., Veith, T., Schneider, J., Beheim-Schwarzbach, J., Bleicker, T., Tesch, J., Schmidt, M. L., Sander, L. E., Kurth, F., Menzel, P., Schwarzer, R., Zuchowski, M., Hofmann, J., Krumbholz, A., Stein, A., Edelmann, A., Corman, V. M., \& Drosten, C. (2021). Estimating infectiousness throughout SARS-CoV-2 infection course. Science, 373(6551). https://doi.org/10.1126/science.abi5273

Kelly, J. A., Amirkhanian, Y. A., Seal, D. W., Galletly, C. M., DiFranceisco, W., Glasman, L. R., Stevenson, L. Y., \& Rosado, N. (2010). Levels and predictors of sexual HIV risk in social networks of men who have sex with men in the Midwest. AIDS Education and Prevention, 22(6), 483-495. https://doi.org/10.1521/aeap.2010.22.6.483

Kermack, W., \& McKendrick, A. (1927). A contribution to the mathematical theory of epidemics. Proceedings of the Royal Society A, 115(772), 700-721.

Ladoy, A., Opota, O., Carron, P. N., Guessous, I., Vuilleumier, S., Joost, S., \& Greub, G. (2021). Size and duration of COVID-19 clusters go along with a high SARS-CoV-2 viral load: A spatio-temporal investigation in Vaud state, Switzerland. Science of the Total Environment, 787, 147483. https://doi.org/10.1016/j.scitotenv.2021.147483

Liu, Y., Eggo, R. M., \& Kucharski, A. J. (2020). Secondary attack rate and superspreading events for SARS-CoV-2. The Lancet, 395(10227), e47. https://doi.org/10.1016/S0140-6736(20)30462-1

Lloyd-Smith, J. O., Schreiber, S. J., Kopp, P. E., \& Getz, W. M. (2005). Superspreading and the effect of individual variation on disease emergence. Nature, 438(7066), 355359. https://doi.org/10.1038/nature04153 
Ma, J., \& Earn, D. J. D. (2006). Generality of the final size formula for an epidemic of a newly invading infectious disease. Bulletin of Mathematical Biology, 68(3), 679702. https://doi.org/10.1007/s11538-005-9047-7

Marks, M., Millat-Martinez, P., Ouchi, D., Roberts, C. H., Alemany, A., Corbacho-Monné, M., Ubals, M., Tobias, A., Tebé, C., Ballana, E., Bassat, Q., Baro, B., Vall-Mayans, M., GBeiras, C., Prat, N., Ara, J., Clotet, B., \& Mitjà, O. (2021). Transmission of COVID-19 in 282 clusters in Catalonia, Spain: a cohort study. The Lancet Infectious Diseases, 21(5), 629-636. https://doi.org/10.1016/S1473-3099(20)30985-3

Miller, D., Martin, M. A., Harel, N., Tirosh, O., Kustin, T., Meir, M., Sorek, N., Gefen-Halevi, S., Amit, S., Vorontsov, O., Shaag, A., Wolf, D., Peretz, A., Shemer-Avni, Y., RoifKaminsky, D., Kopelman, N. M., Huppert, A., Koelle, K., \& Stern, A. (2020). Full genome viral sequences inform patterns of SARS-CoV-2 spread into and within Israel. Nature Communications, 11(1). https://doi.org/10.1038/s41467-02019248-0

Mumford, J., Hannant, D., \& Jessett, D. (1990). Experimental infection of ponies with equine influenza (H3N8) viruses by intranasal inoculation or exposure to aerosols. Equine Veterinary Journal, 22, 93-98.

Omisakin, F., MacRae, M., Ogden, I. D., \& Strachan, N. J. C. (2003). Concentration and prevalence of Escherichia coli 0157 in cattle feces at slaughter. Applied and Environmental Microbiology, 69(5), 2444-2447. https://doi.org/10.1128/AEM.69.5.2444-2447.2003

R Core Team. (2020). $R$ : A language and environment for statistical computing. $R$ Foundation for Statistical Computing. https://www.r-project.org/

Singanayagam, A., Hakki, S., Dunning, J., Madon, K. J., Crone, M. A., Koycheva, A., Derquifernandez, N., Barnett, J. L., Whitfield, M. G., Varro, R., Charlett, A., Kundu, R., Fenn, J., Cutajar, J., Quinn, V., Conibear, E., Barclay, W., \& Freemont, P. S. (2021). Community transmission and viral load kinetics of the SARS-CoV-2 delta (B.1.617.2) variant in vaccinated and unvaccinated individuals in the UK: a prospective, longitudinal, cohort study. Lancet, 3099(21). https://doi.org/10.1016/S1473-3099(21)00648-4 Soetaert, K., Petzoldt, T., \& Setzer, R. W. (2010). Solving differential equations in R: Package deSolve. Journal of Statistical Software, 33(9), 1-25. https://doi.org/10.18637/jss.v033.i09

Stein, R. A. (2011). Super-spreaders in infectious diseases. International Journal of Infectious Diseases, 15(8), e510-e513. https://doi.org/10.1016/j.ijid.2010.06.020

Strong, R., la Rocca, S. A., Paton, D., Bensaude, E., Sandvik, T., Davis, L., Turner, J., Drew, T., Raue, R., Vangeel, I., \& Steinbach, F. (2015). Viral dose and immunosuppression modulate the progression of acute BVDV-1 infection in calves: Evidence of long term persistence after intra-nasal infection. PLoS ONE, 10(5), 1-13. https://doi.org/10.1371/journal.pone.0124689

Taube, J. C., Miller, P. B., \& Drake, J. M. (2021). An open-access database of infectious disease transmission trees to explore superspreader epidemiology. MedRxiv, 2021.01.11.21249622. https://doi.org/10.1101/2021.01.11.21249622

van Damme, W., Dahake, R., van de Pas, R., Vanham, G., \& Assefa, Y. (2021). COVID-19: Does the infectious inoculum dose-response relationship contribute to understanding heterogeneity in disease severity and transmission dynamics? Medical Hypotheses, 146, 110431. https://doi.org/10.1016/j.mehy.2020.110431

Woolhouse, M. E. J., Dye, C., Etard, J. F., Smith, T., Charlwood, J. D., Garnett, G. P., Hagan, P., Hii, J. L. K., Ndhlovu, P. D., Quinnell, R. J., Watts, C. H., Chandiwana, S. K., \& Anderson, R. M. (1997). Heterogeneities in the transmission of infectious agents. 
Proceedings of the National Academy of Sciences of the United States of America, 94, 338-342.

Wu, P., Liu, F., Chang, Z., Lin, Y., Ren, M., Zheng, C., Li, Y., Peng, Z., Qin, Y., Yu, J., Geng, M., Yang, X., Zhao, H., Li, Z., Zhou, S., Ran, L., Cowling, B. J., Lai, S., Chen, Q., ... Li, Z. (2021). Assessing Asymptomatic, Presymptomatic, and Symptomatic Transmission Risk of Severe Acute Respiratory Syndrome Coronavirus 2. Clinical Infectious Diseases, 73(6), e1314-e1320. https://doi.org/10.1093/cid/ciab271

Zarkov, I., \& Bochev, I. (2008). Influence of inoculation dose of avian H6N2 influenza A virus on virus shedding and humoral immune response of chickens after artificial experimental intravenous infection. Revue de Medecine Veterinaire, 159(10), 489493.

Zhang, Y., Li, Y., Wang, L., Li, M., \& Zhou, X. (2020). Evaluating transmission heterogeneity and super-spreading event of COVID-19 in a metropolis of China. International Journal of Environmental Research and Public Health, 17(10). https://doi.org/10.3390/ijerph17103705

Zhou, L., Ayeh, S. K., Chidambaram, V., \& Karakousis, P. C. (2021). Modes of transmission of SARS-CoV-2 and evidence for preventive behavioral interventions. BMC Infectious Diseases, 21(1), 1-9. https://doi.org/10.1186/s12879-021-06222-4 


\section{Supplementary Files}

This is a list of supplementary files associated with this preprint. Click to download.

- WaneliketalSupplementary.pdf 